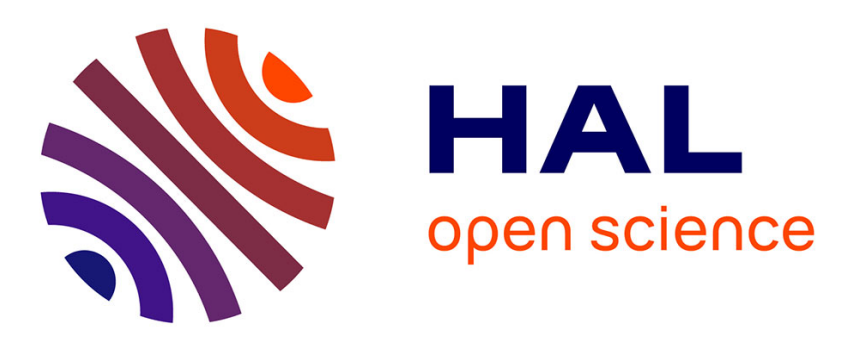

\title{
Multiobjective Design Optimization of 3-PRR Planar Parallel Manipulators
}

Raza Ur-Rehman, Stéphane Caro, Damien Chablat, Philippe Wenger

\section{To cite this version:}

Raza Ur-Rehman, Stéphane Caro, Damien Chablat, Philippe Wenger. Multiobjective Design Optimization of 3-PRR Planar Parallel Manipulators. 20th CIRP Design conference, Apr 2010, Nantes, France. 10.1007/978-3-642-15973-2_37. hal-00464101

\section{HAL Id: hal-00464101 https://hal.science/hal-00464101}

Submitted on 16 Mar 2010

HAL is a multi-disciplinary open access archive for the deposit and dissemination of scientific research documents, whether they are published or not. The documents may come from teaching and research institutions in France or abroad, or from public or private research centers.
L'archive ouverte pluridisciplinaire HAL, est destinée au dépôt et à la diffusion de documents scientifiques de niveau recherche, publiés ou non, émanant des établissements d'enseignement et de recherche français ou étrangers, des laboratoires publics ou privés. 


\title{
Multiobjective Design Optimization of $3-\underline{P} R R$ Planar Parallel Manipulators
}

\author{
Raza UR-REHMAN, Stéphane CARO, Damien CHABLAT, Philippe WENGER \\ Institut de Recherche en Communications et Cybernétique de Nantes \\ UMR CNRS 6597, 1 rue de la Noë, 44321 Nantes, France \\ \{ur-rehman, caro, chablat, wenger\}@irccyn.ec-nantes.fr
}

\begin{abstract}
This paper addresses the dimensional synthesis of parallel kinematics machines. A multiobjective optimization problem is proposed in order to determine optimum structural and geometric parameters of parallel manipulators. The proposed approach is applied to the optimum design of a three-degree-of-freedom planar parallel manipulator in order to minimize the mass of the mechanism in motion and to maximize its regular shaped workspace.
\end{abstract}

Keywords: Multiobjective Optimization, Parallel Manipulators, Workspace, Design.

\section{INTRODUCTION}

The design of parallel kinematics machines is a complex subject. The fundamental problem is that their performance heavily depends on their geometry [1] and the mutual dependency of almost all the performance measures. This makes the problem computationally complex and yields the traditional solution approaches inefficient. As reported in [2], since the performance of a parallel manipulator depends on its dimensions, the latter depend on the manipulator application(s). Furthermore, numerous design aspects contribute to the Parallel Kinematics Machine (PKM) performance and an efficient design will be one that takes into account all or most of these design aspects. This is an iterative process and an efficient design requires a lot of computational efforts and capabilities for mapping design parameters into design criteria, and hence turning out with a multiobjective design optimization problem. Indeed, the optimal geometric parameters of a PKM can be determined by means of a the resolution of a multiobjective optimization problem. The solutions of such a problem are nondominated solutions, also called Pareto-optimal solutions. Therefore, design optimization of parallel mechanisms is a key issue for their development.

Several researchers have focused on the optimization problem of parallel mechanisms the last few years. They have come up either with mono- or multi-objective design optimization problems. For instance, Lou et al. presented a general approach for the optimal design of parallel manipulators to maximize the volume of an effective regular-shaped workspace while subject to constraints on their dexterity [3, 4]. Hay and Snyman [1] considered the optimal design of parallel manipulators to obtain a prescribed workspace, whereas Ottaviano and Ceccarelli [5, 6] proposed a formulation for the optimum design of 3-Degree-Of-Freedom (DOF) spatial parallel manipulators for given position and orientation workspaces. They based their study on the static analysis and the singularity loci of a manipulator in order to optimize the geometric design of the Tsai manipulator for a given free from singularity workspace. Hao and Merlet [7] discussed a multi-criterion optimal design methodology based on interval analysis to determine the possible geometric parameters satisfying two compulsory requirements of the workspace and accuracy. Similarly, Ceccarelli et al. \& dealt with the multi-criterion optimum design of both parallel and serial manipulators with the focus on the workspace aspects, singularity and stiffness properties. Gosselin and Angeles 19, 10 analyzed the design of a 3-DOF planar and a 3-DOF spherical parallel manipulators by maximizing their workspace volume while paying attention to their dexterity. Pham and Chen [11] suggested maximizing the workspace of a parallel flexure mechanism with the constraints on a global and uniformity measure of manipulability. Stamper et al. 12 used the global conditioning index based on the integral of the inverse condition number of the kinematic Jacobian matrix over the workspace in order to optimize a spatial 3-DOF translational parallel manipulator. Stock and Miller 13 formulated a weighted sum multi-criterion optimization problem with manipulability and workspace as two objective functions. Menon et al. 14 used the maximization of the first natural frequency as an objective function for the geometrical optimization of the parallel mechanisms. Similarly, Li et al. 15 proposed dynamics and elastodynamics optimization of a 2-DOF planar parallel robot to improve the dynamic accuracy of the mechanism. They proposed a dynamic index to identify the range of natural frequency with different configurations. Krefft [16 also formulated a multi-criterion elastodynamic optimization problem for parallel mechanisms while considering workspace, velocity transmission, inertia, stiffness and the first natural frequency as optimization objectives. Chablat and Wenger [17 proposed an analytical approach for the architectural optimization of a 3-DOF transla- 
tional parallel mechanism, named Orthoglide 3-axis, based on prescribed kinetostatic performance to be satisfied in a given Cartesian workspace.

Most of the foregoing research works aimed to improve the mechanisms' performance throughout their whole workspace. In this paper, the mechanisms' performance are improved over a regular shaped workspace that is defined based on the specifications. As a result, we propose a methodology to deal with the multiobjective design optimization of PKMs. The size of the regular shaped workspace and the mass in motion of the mechanism are the objective functions of the optimization problem. Its constraints are determined based on the mechanism accuracy, assembly and the conditioning number of its kinematic Jacobian matrix. The proposed approach is highlighted with the optimal design of a 3-DOF Planar Parallel Manipulator (PPM). The non-dominated solutions, also called Pareto-optimal solutions, are obtained by means of a genetic algorithm.

\section{$23-\underline{P} R R$ PLANAR PARALLEL MANIPU- LATORS}

Planar parallel manipulators are distinguished by the fact that all their components and corresponding motions, including their end-effector, generate planar motions. Their architecture is simple and they are usually simple to control. They can find many applications in planar motions that require high dynamics. Their weakness is their difficulty to carry out a large payload whose the weight is normal to the plane of motion. 3-DOF PPMs are classified in families, namely, the 3-RRR, 3-RPR, 3-PRR and 3-PPP PPMs where $R$ and $P$ stand for revolute and prismatic joints, respectively. Those families are described in [2]. In the scope of this paper, we focus on the optimum design of 3- $\underline{P} P P$ PPMs, where $\underline{P}$ denotes an actuated prismatic joint. However, the proposed approach can be applied to any type of PPM.

\subsection{Manipulator architecture}

A 3-DOF PPM with three identical chains is shown in Fig. 1. Each kinematic chain is of $\underline{P} R R$-type and consists of one actuated prismatic joint, $\underline{P}$; two revolute joints, $R$; and two links. This $3-\underline{P} R R$ manipulator is intended to position and orient the equilateral triangle-shaped platform $C_{1} C_{2} C_{3}$ in the plane of motion. The geometric center of platform $C_{1} C_{2} C_{3}$, denoted by $P$, is the operation point of the manipulator. The displacements of the three prismatic joints, i.e., $\rho_{1}, \rho_{2}$ and $\rho_{3}$, are the input variables whereas the Cartesian coordinates of point $P$, i.e., $x_{p}$ and $y_{p}$, and the orientation $\phi$ of the platform are the output variables. The base-platform of the manipulator is also an equilateral triangle with vertices $A_{1}, A_{2}$ and $A_{3}$, point $O$ is its geometric center and the origin of the reference frame. The prismatic actuators are aligned to its sides and are attached to points $A_{i}(i=1,2,3)$ with orientation angles $\alpha_{1}, \alpha_{2}$ and $\alpha_{3}$ being equal to $0^{\circ}$, $120^{\circ}$ and $240^{\circ}$, respectively. Here are the parameters describing the manipulator geometry:
- $R$ : radius of the circumscribed circle of triangle $A_{1} A_{2} A_{3}$ of center $O$, i.e., $R=O A_{i}$;

- $r$ : radius of the circumscribed circle of triangle $C_{1} C_{2} C_{3}$ of center $P$, i.e., $r=P C_{i}$;

- $L_{b}$ : the length of the intermediate links, i.e., $L_{b}=$ $B_{i} C_{i}$

- $r_{j}$ : the cross-section radius of the intermediate link;

- $r_{p}$ : the cross-section radius of the moving platform link.

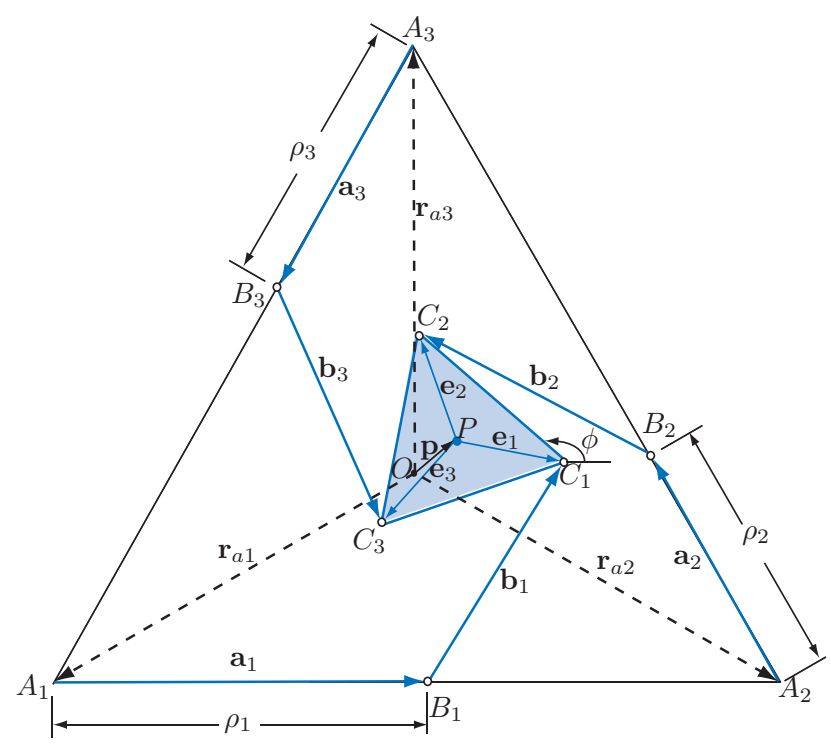

Figure 1: $3-\underline{P} R R$ planar parallel manipulator

\subsection{Kinematic modeling of the $3-\underline{P} R R$ PPM}

Knowing the geometric parameters of the mechanism, i.e., $R, r$ and $L_{b}$, its Inverse Kinematics Model (IKM) gives the relation between the actuators displacements $\rho_{i}$ and the moving platform pose, i.e., $x_{p}, y_{p}$ and $\phi$ :

$\boldsymbol{\rho}=f\left(\mathbf{x}_{p}\right)$

with

$$
\begin{aligned}
\boldsymbol{\rho} & =\left[\begin{array}{lll}
\rho_{1} & \rho_{2} & \rho_{3}
\end{array}\right]^{T} \\
\mathbf{x}_{p} & =\left[\begin{array}{lll}
x_{p} & y_{p} & \phi
\end{array}\right]^{T}
\end{aligned}
$$

Eq. (11) can be expressed as a quadratic equation 18. The latter may have eight solutions corresponding to the eight working modes of the mechanism [19]. The choice of the working mode can also be used as a design parameter of the mechanism as it modifies the location of its singular configurations. The Direct Kinematics Model (DKM) of the manipulator characterizes the moving platform pose in terms of the prismatic actuators displacements:

$\mathbf{x}_{p}=f(\boldsymbol{\rho})$

The DKM of the $3-\underline{P} R R$ PPM may have six solutions corresponding to the six assembly modes of the mechanism 20]. 


\subsection{Kinematic Jacobian matrix of the $3-\underline{P} R R$ PPM}

The kinematic Jacobian matrix defines the relationship between the actuators and mobile platform velocity vectors. For the $i^{\text {th }}$ kinematic chain, a closed loop vector equation can be written as:

$\overrightarrow{O P}=\overrightarrow{O A_{i}}+\overrightarrow{A_{i} B_{i}}+\overrightarrow{B_{i} C_{i}}+\overrightarrow{C_{i} P}$

Equation (4) can be expressed algebraically as:

$\mathbf{p}=R \mathbf{r}_{a i}+\rho_{i} \mathbf{a}_{i}+L_{b} \mathbf{b}_{i}-r \mathbf{e}_{i}$

with $\mathbf{a}_{i}, \mathbf{b}_{i}, \mathbf{e}_{i}$ and $\mathbf{r}_{a i}$ being the unit vectors depicted in Fig. 1. Upon differentiation of Eq. (5) with respect to time we get,

$\dot{\mathbf{p}}=\dot{\rho}_{i} \mathbf{a}_{i}+L_{b} \dot{\mathbf{b}}_{i}-r \dot{\mathbf{e}}_{i}$

$\dot{\mathbf{b}}_{i}$ and $\dot{\mathbf{e}}_{i}$ being written as:

$\dot{\mathbf{b}}_{i}=\dot{\beta}_{i} \mathbf{E b}_{i} \quad \dot{\mathbf{e}}_{i}=\dot{\phi} \mathbf{E} \mathbf{e}_{i}$

$\dot{\beta}_{i}$ is the angular velocity of the $i^{t h}$ intermediate link and $\mathbf{E}$ is the right angle rotation matrix given by,

$\mathbf{E}=\left[\begin{array}{cc}0 & -1 \\ 1 & 0\end{array}\right]$

Accordingly, Eq. (6) becomes

$\dot{\mathbf{p}}=\dot{\rho}_{i} \mathbf{a}_{i}+L_{b} \dot{\beta}_{i} \mathbf{E b}_{i}-r \dot{\phi} \mathbf{E} \mathbf{e}_{i}$

Upon multiplication of Eq. (9) by $\mathbf{b}_{i}^{T}$, we obtain the matrix form:

$\mathbf{A} \dot{\mathbf{x}}_{p}=\mathbf{B} \dot{\rho}$

with

$\mathbf{A}=\left[\begin{array}{cc}\mathbf{b}_{1}^{T} & r \mathbf{b}_{1}^{T} \mathbf{E e}_{1} \\ \mathbf{b}_{2}^{T} & r \mathbf{b}_{2}^{T} \mathbf{E e}_{2} \\ \mathbf{b}_{3}^{T} & r \mathbf{b}_{3}^{T} \mathbf{E e}_{3}\end{array}\right]$

and

$\mathbf{B}=\left[\begin{array}{ccc}\mathbf{b}_{1}^{T} \mathbf{a}_{1} & 0 & 0 \\ 0 & \mathbf{b}_{2}^{T} \mathbf{a}_{2} & 0 \\ 0 & 0 & \mathbf{b}_{3}^{T} \mathbf{a}_{3}\end{array}\right]$

Therefore, the prismatic joints rates are expressed in terms of the moving platform twist as follows:

$\dot{\boldsymbol{\rho}}=\mathbf{B}^{-1} \mathbf{A} \dot{\mathbf{x}}_{p}=\mathbf{J} \dot{\mathbf{x}}_{p}$

where $\mathbf{J}$ is the kinematic Jacobian matrix of the manipulator.

$\mathbf{J}=\mathbf{B}^{-1} \mathbf{A}=\frac{1}{\mathbf{a}_{i} \cdot \mathbf{b}_{i}}\left[\begin{array}{ll}\mathbf{b}_{1} & r \mathbf{k}\left(\mathbf{b}_{1} \times \mathbf{e}_{1}\right) \\ \mathbf{b}_{2} & r \mathbf{k}\left(\mathbf{b}_{2} \times \mathbf{e}_{2}\right) \\ \mathbf{b}_{3} & r \mathbf{k}\left(\mathbf{b}_{3} \times \mathbf{e}_{3}\right)\end{array}\right]$

The singular configurations of the $3-\underline{P} R R$ PPM can be obtained by means of a singularity analysis of $\mathbf{J}$ as explained in [18].

\subsection{Stiffness matrix}

The stiffness model of the $3-\underline{P} R R$ PPM is obtained by means of the refined lumped mass modeling presented in 21 .

Let us consider a general schematic of the $3-\underline{P} R R$ $\mathrm{PPM}$ that is composed of a mobile platform connected to a fixed base by means of three identical kinematics chains, as shown in Fig. 2. Each kinematic contains an actuated prismatic joint and two passive revolute joints.

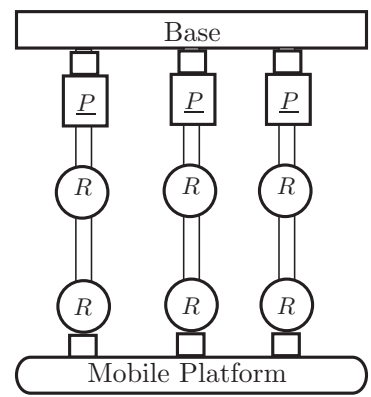

Figure 2: Schematic diagram of a $3-\underline{P} R R$

According to the flexible model described in 21, each kinematic chain of the $3-\underline{P} R R$ manipulator can be considered as a serial architecture as shown in Fig. 3 that contains sequentially:

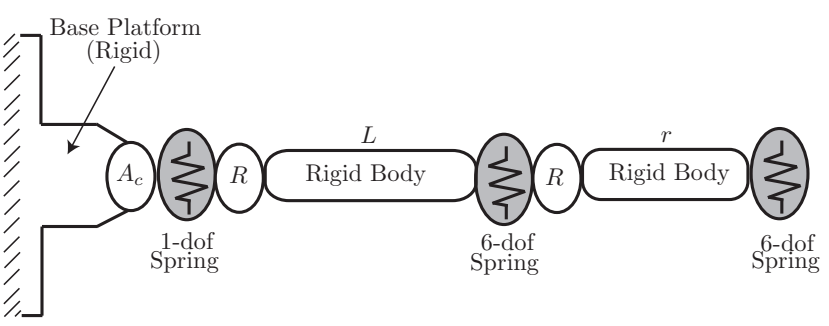

Figure 3: Flexible model of the single kinematic chains of the $3-\underline{P} R R$ PPM, $A_{c}$ stands for actuating joint and $R$ for revolute joint

- a rigid link between the manipulator base and the $i^{\text {th }}$ actuated joint (part of the base platform) described by the constant homogeneous transformation matrix $\mathbf{T}_{\text {Base; }}^{i}$

- a 1-dof actuated joint, defined by the homogeneous matrix function $\mathbf{V}_{a}\left(q_{0}^{i}\right)$ where $q_{0}^{i}$ is the actuated coordinate;

- a 1-dof virtual spring describing the actuator mechanical stiffness, which is defined by the homogeneous matrix function $\mathbf{V}_{s 1}\left(\theta_{0}^{i}\right)$ where $\theta_{0}^{i}$ is the virtual spring coordinate corresponding to the translational spring;

- a 1-dof passive $R$-joint at the beginning of the leg allowing one rotation angle $q_{2}^{i}$, which is described by the homogeneous matrix function $\mathbf{V}_{r 1}\left(q_{2}^{i}\right)$

- a rigid leg of length $L$ linking the foot and the movable platform, which is described by the constant homogeneous transformation matrix $\mathbf{T}_{L}^{i}$; 
- a 6-dof virtual spring describing the leg stiffness, which is defined by the homogeneous matrix function $\mathbf{V}_{s 2}\left(\theta_{1}^{i} \cdots \theta_{6}^{i}\right)$, with $\theta_{1}^{i}, \theta_{2}^{i}, \theta_{3}^{i}$ and $\theta_{4}^{i}, \theta_{5}^{i}, \theta_{6}^{i}$ being the virtual spring coordinates corresponding to the spring translational and rotational deflections;

- a 1-dof passive $R$-joint between the leg and the platform, allowing one rotation angle $q_{3}^{i}$, which is described by the homogeneous matrix function $\mathbf{V}_{r 2}\left(q_{3}^{i}\right)$;

- a rigid link of length $r$ from the manipulator leg to the geometric center of the mobile platform, which is described by the constant homogeneous transformation matrix $\mathbf{T}_{r}^{i}$;

- a 6-dof virtual spring describing the stiffness of the moving platform, which is defined by the homogeneous matrix function $\mathbf{V}_{s 3}\left(\theta_{7}^{i} \cdots \theta_{12}^{i}\right), \theta_{7}^{i}, \theta_{8}^{i}, \theta_{9}^{i}$ and $\theta_{10}^{i}, \theta_{11}^{i}, \theta_{12}^{i}$ being the virtual spring coordinates corresponding to translational and rotational deflections of link $C_{i} P$;

- a homogeneous transformation matrix $\mathbf{T}_{E n d}^{i}$ characterizing the rotation from the 6 -dof spring associated with link $C_{i} P$ and the manipulator base frame;

The corresponding mathematical expression defining the end-effector location subject to variations in all above defined coordinates of the $i^{\text {th }}$ kinematic chain can be written as follows:

$$
\begin{aligned}
\mathbf{T}^{i}= & \mathbf{T}_{\text {Base }}^{i} \mathbf{V}_{a}^{i}\left(q_{0}^{i}\right) \mathbf{V}_{s 1}\left(\theta_{0}^{i}\right) \mathbf{V}_{r 1}\left(q_{1}^{i}\right) \mathbf{T}_{L}^{i} \mathbf{V}_{s 2} \\
& \left(\theta_{1}^{i} \cdots \theta_{6}^{i}\right) \mathbf{V}_{r 2}\left(q_{2}^{i}\right) \mathbf{T}_{r}^{i} \mathbf{V}_{s 3}\left(\theta_{7}^{i} \cdots \theta_{12}^{i}\right) \mathbf{T}_{\text {Base }}^{i}
\end{aligned}
$$

From 21, the kinetostatic model of the ith leg of the $3-\underline{P} R R \mathrm{PPM}$ can be reduced to a system of two matrix equations, namely,

$$
\left[\begin{array}{cc}
\mathbf{S}_{\theta}^{i} & \mathbf{J}_{q}^{i} \\
\mathbf{J}_{q}^{i} & \mathbf{0}_{2 \times 2}
\end{array}\right]\left[\begin{array}{c}
\mathbf{f}_{i} \\
\delta \mathbf{q}_{i}
\end{array}\right]=\left[\begin{array}{c}
\delta \mathbf{t}_{i} \\
\mathbf{0}_{2}
\end{array}\right]
$$

where the sub-matrix $\mathbf{S}_{\theta}^{i}=\mathbf{J}_{\theta}^{i} \mathbf{K}_{\theta}^{i-1} \mathbf{J}_{\theta}^{i^{T}}$ describes the spring compliance relative to the geometric center of the moving platform, and the sub-matrix $\mathbf{J}_{q}^{i}$ takes into account the passive joint influence on the moving platform motions.

$\mathbf{K}_{\theta}^{i-1}$ matrix, of size $13 \times 13$, describes the compliance of the virtual springs and takes the form:

$$
\mathbf{K}_{\theta}^{i-1}=\left[\begin{array}{ccc}
\mathbf{K}_{a c t}^{i-1} & \mathbf{0}_{1 \times 6} & \mathbf{0}_{1 \times 6} \\
\mathbf{0}_{6 \times 1} & \mathbf{K}_{l e g}^{i}-1 & \mathbf{0}_{6 \times 6} \\
\mathbf{0}_{6 \times 1} & \mathbf{0}_{6 \times 6} & \mathbf{K}_{p f}^{i}{ }^{-1}
\end{array}\right]
$$

where $\mathbf{K}_{a c t}^{i}$ is the $1 \times 1$ stiffness matrix of the ith actuator, $\mathbf{K}_{\text {leg }}^{i}$ is the $6 \times 6$ stiffness matrix of the ith intermediate leg and $\mathbf{K}_{p f}^{i}$ is the $6 \times 6$ the stiffness matrix of the ith link of the moving platform. The compliance matrices of the intermediate legs and the ith link of the moving platform are calculated by means of the stiffness model of a cantilever beam, namely,

$$
\mathbf{K}_{L}^{i}{ }^{-1}=\left[\begin{array}{cccccc}
\frac{L}{E A} & 0 & 0 & 0 & 0 & 0 \\
0 & \frac{L^{3}}{3 E I_{z}} & 0 & 0 & 0 & \frac{L^{2}}{2 E I_{z}} \\
0 & 0 & \frac{L^{3}}{3 E I_{y}} & 0 & -\frac{L^{2}}{2 E I_{y}} & 0 \\
0 & 0 & 0 & \frac{L}{G I_{x}} & 0 & 0 \\
0 & 0 & -\frac{L^{2}}{2 E I_{y}} & 0 & \frac{L}{E I_{y}} & 0 \\
0 & \frac{L^{2}}{2 E I_{z}} & 0 & 0 & 0 & \frac{L}{E I_{z}}
\end{array}\right]
$$

$L$ is the length of the beam, i.e., $L=L_{b}$ for the intermediate legs and $L=r$ for the moving platform links. $A$ is the cross-sectional area of the beam, i.e., $A_{L_{b}}=\pi r_{j}^{2}$ and $A_{r}=\pi r_{p}^{2} . I_{z}=I_{y}$ is the polar moment of inertia about $y$ and $z$ axes, i.e., for the intermediate legs and the moving platform links, their expressions are $\pi r_{j}^{4} / 4$ and $\pi r_{p}^{4} / 4$, respectively. $I_{x}=I_{z}+I_{y}$ is the polar moment of inertia about the longitudinal axis of the beam. $E$ is the Young modulus of the material and $G$ its shear modulus

$\mathbf{J}_{\theta}^{i}$ of size $6 \times 13$ is the Jacobian matrix related to the virtual springs and $\mathbf{J}_{q}^{i}$ of size $6 \times 2$, the one related to the passive joints. $\mathbf{f}_{i}$ is the wrench exerted on the ith leg of the $3-\underline{P} R R$ PPM at the geometric center of the moving platform and $\delta \mathbf{t}_{i}$ is the corresponding translational and rotational displacements vector.

Therefore, the Cartesian stiffness matrix $\mathbf{K}_{i}$ of the ith leg defining the motion-to-force mapping is obtained from Eq. (16).

$\mathbf{f}_{i}=\mathbf{K}_{i} \delta \mathbf{t}_{i}$

Finally, the Cartesian stiffness matrix $\mathbf{K}$ of the $3-\underline{P} R R$ PPM is found with a simple addition of $\mathbf{K}_{i}$ matrices, namely,

$$
\mathbf{K}=\sum_{i=1}^{3} \mathbf{K}_{i}
$$

\section{MULTIOBJECTIVE DESIGN OPTI- MIZATION}

In general, the design process of PKMs simultaneously deals with two groups of criteria, one relates to the kinematic properties while the other relates to the kinetostatic/dynamic properties of the mechanism. Both of these groups include a number of performance measures that essentially vary throughout the workspace but remain within the prescribed bounds. Kinematic aspects are comparatively less complex and are usually based on the concept of critical points whereas kinetostatic aspects work with a detailed description of the structure and their evaluation is usually time consuming. Indeed, one of the major design issues in kinetostatic design is the computation of the stiffness matrix 22. Accordingly, a multiobjective design optimization approach is proposed based on performance measures/criteria from both kinematic and kinetostatic domains. On the one hand, this approach deals with the geometric/kinematic design in order to determine the PKM geometry including the link 
lengths and the joint limits. On the other hand, it considers the kinetostatic design to determine the size and the mass properties of the links.

\subsection{Optimization objectives}

The multiobjective optimization problem aims to determine the optimum geometric parameters of a PKM in order to maximize its workspace as well as to minimize the mass of the mechanism in motion. Here, the workspace of the mechanism is discretized and the considered performance measures and constraints are evaluated and verified for each point.

\subsubsection{Mass in motion of the mechanism}

The mass in motion of the mechanism is considered to be the first objective function of the optimization problem. Mass and inertia are functions of manipulator dimensions, i.e., link lengths, cross-sectional area, thickness. Hence, in general, the mass in motion $m_{t}$ of the mechanism is composed of the mass of the platform, $m_{p f}$, the mass of the three intermediate bars, $m_{b}$, and the mass in motion of the three prismatic actuators, $m_{s}$ :

$m_{t}=m_{p f}+3 m_{b}+3 m_{s}$

Since the actuators are fixed, their mass is considered to be constant while the mass of the other two components can easily be calculated by using the geometry of the components and the density $d$ of their material, given as,

$m_{p f}=\pi r_{p}^{2} r d, \quad m_{b}=\pi r_{j}^{2} L_{b} d$

Consequently, the first objective function of the optimization problem is written as:

$f_{1}(\mathbf{x})=m_{t} \rightarrow \min$

$\mathbf{x}$ being the vector of the geometric design parameters of the mechanism.

\subsubsection{Regular workspace size}

Workspace is one of the most important design issues as it defines the working volume of the robot/manipulator and determines the area that can be reached by a reference frame located on the moving platform or end-effector 23, 12]. The size and shape of the workspace are of primary importance for the global geometric performance evaluations of the manipulators [24].

The quality of the workspace that reflects the shape, size, presence of singularities is of prime importance in PKM design. Workspace based design optimization of parallel mechanisms can usually be solved with two different formulations. The first formulation aims to design a manipulator whose workspace contains a prescribed workspace and the second approach aims to design a manipulator, of which the workspace is as large as possible. However, maximizing the workspace may result in poor design with regard to the manipulator dexterity and manipulability 12. This problem can be solved by properly defining the constraints of the optimization problem. Here, the multiobjective optimization problem of PKMs is based on the formulation of workspace maximization, i.e, to determine the optimum geometry of PKM in order to maximize a regular-shaped workspace. Workspace size can be defined by its geometric shape parameters like the radius of a cylindrical/spherical workspace or the sides of the cube for a cubic workspace.

In the scope of the paper, a cylindrical workspace defined with its radius $R_{w}$ is considered. Furthermore, at each point of the workspace, an angular rotation range $\Delta \phi=20^{\circ}$ of the platform about the $Z$-axis can be achieved. A 3-dimensional schematic of the regular shaped workspace is shown in Fig. A, where $x_{c}, y_{c}$ are the coordinates of the center of the regular dextrous workspace and $\phi_{c}$ is the orientation of the platform at its home-posture (see Fig. 11).

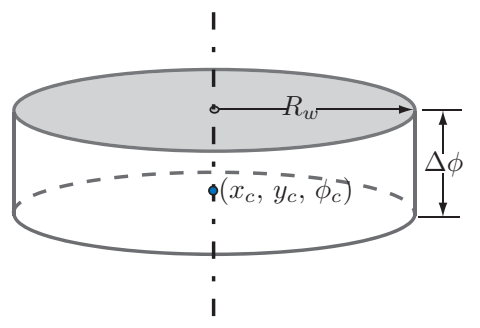

Figure 4: $3-\underline{P} R R$ workspace

Consequently, in order to maximize the manipulator workspace, the second objective of the optimization problem can be written as:

$f_{2}(\mathbf{x})=R_{w} \rightarrow \max$

\subsection{Optimization constraints}

Besides, the geometric and actuator constraints of the PKM, conditioning of the kinematic Jacobian matrix and accuracy obtained from the stiffness characteristics of the mechanism are considered. Constraining the conditioning of the Jacobian matrix guarantees singularity free workspace whereas limits on accuracy consideration ensure sufficient mechanism stiffness.

\subsubsection{Geometric Constraints}

The first constraint is related to the mechanism assembly, namely,

$L_{b}+r \geq R / 2$

In order to avoid intersections between prismatic joints, the lower and upper bounds of the prismatic lengths are defined as follows:

$0<\rho_{i}<\sqrt{3} R$

\subsubsection{Condition number of the kinematic Jacobian matrix}

The condition number $\kappa_{F}(\mathbf{M})$ of a $m \times n$ matrix $\mathbf{M}$, with $m \leq n$, based on the Frobenius norm is defined as follows,

$\kappa_{F}(\mathbf{M})=\frac{1}{m} \sqrt{\operatorname{tr}\left(\mathbf{M}^{T} \mathbf{M}\right) \operatorname{tr}\left[\left(\mathbf{M}^{T} \mathbf{M}\right)^{-1}\right]}$ 
Here, the condition number is computed based on the Frobenius norm as the latter produces a condition number that is analytic in terms of the posture parameters whereas the 2-norm does not. Besides, it is much costlier to compute singular values than to compute matrix inverses.

The terms of the direct Jacobian matrix of the 3$\underline{P} R R$ PPM are not homogeneous as they do not have same units. Accordingly, its condition number is meaningless. Indeed, its singular values cannot be arranged in order as they are of different nature. However, from [25] and 26], the Jacobian can be normalized by means of a normalizing length. Later on, the concept of characteristic length was introduced in 27] in order to avoid the random choice of the normalizing length. For instance, the previous concept was used in [18 to analyze the kinetostatic performance of manipulators with multiple inverse kinematic solutions, and therefore to select their best working mode.

Accordingly, for the design optimization of 3 $\underline{P} R R$ PPM, the minimum of the inverse condition number of the kinematic Jacobian matrix, $\kappa^{-1}(\mathbf{J})$, is supposed to be higher than a prescribed value, say 0.1 , throughout the manipulator workspace, for any rotation of its end-effector, i.e.,

$\min \left(\kappa^{-1}(\mathbf{J})\right) \geq 0.1$

\subsubsection{Accuracy constraints}

The position and orientation accuracy is assessed by using the stiffness parameters of the mechanism. Let $(\delta x, \delta y, \delta z)$ and $\left(\delta \phi_{x}, \delta \phi_{y}, \delta \phi_{z}\right)$ be the position and orientation errors of the end-effector subjected to external forces $\left(F_{x}, F_{y}, F_{z}\right)$ and torques $\left(\tau_{z}, \tau_{y}, \tau_{z}\right)$. The constraints related to the accuracy of the manipulator are defined as follows:

$$
\begin{aligned}
& \delta x \leq \delta x^{\max } \quad \delta y \leq \delta y^{\max } \quad \delta z \leq \delta z^{\max } \\
& \delta \phi_{x} \leq \delta \phi_{x}^{\max } \quad \delta \phi_{y} \leq \delta \phi_{y}^{\max } \quad \delta \phi_{z} \leq \delta \phi_{z}^{\max }
\end{aligned}
$$

$\left(\delta x^{\max }, \delta y^{\max }, \delta z^{\max }\right)$ being the maximum allowable position errors and $\left(\delta \phi_{x}^{\max }, \delta \phi_{y}^{\max }, \delta \phi_{z}^{\max }\right)$ the maximum allowable orientation errors of the end-effector. These accuracy constraints can be expressed in terms of the components of the mechanism stiffness matrix and the wrench applied to the end-effector. Let us assume that the accuracy requirements are:

$$
\begin{aligned}
\sqrt{\delta x^{2}+\delta y^{2}} & \leq 0.0001 \mathrm{~m} \\
\delta z & \leq 0.001 \mathrm{~m} \\
\delta \phi_{z} & \leq 1 \mathrm{deg}
\end{aligned}
$$

If the end-effector is subjected to a wrench, whose components are $\left\|F_{x, y}\right\|=F_{z}=100 \mathrm{~N}$ and $\tau_{z}=100 \mathrm{Nm}$, then the accuracy constraints can be expressed as:

$$
\begin{aligned}
& k_{x y}^{\text {min }} \geq\left\|F_{x, y}\right\| / \sqrt{\delta x^{2}+\delta y^{2}}=10^{6} \mathrm{~N} \cdot \mathrm{m}^{-1} \\
& k_{z}^{\text {min }} \geq F_{z} / \delta z=10^{5} \mathrm{~N} \cdot \mathrm{m}^{-1} \\
& k_{\phi_{z}}^{\text {min }} \geq \tau_{z} / \delta \phi_{z}=\frac{10}{\pi / 180} \mathrm{~N} \cdot \mathrm{m} \cdot \mathrm{rad}^{-1}
\end{aligned}
$$

\subsection{Design Variables}

Along with the above mentioned geometric parameters $\left(R, r, L_{b}\right)$ of the $3-\underline{P} R R$ PPM, the dimension of the circular-cross-section of the intermediate bars defined with radius $r_{j}$ and the circular-cross-section of the platform bars defined with $r_{p}$ are considered as design variables, also called decision variables. The platform is assumed to be made up of three circular bars, each of length $r$. Hence, the design parameters vector $\mathbf{x}$ is given by:

$\mathbf{x}=\left[\begin{array}{lllll}R & r & L_{b} & r_{j} & r_{p}\end{array}\right]^{T}$

\subsection{Multiobjective optimization problem statement}

The Multiobjective Optimization Problem (MOO) for a $3-\underline{P} R R$ PPM can be stated as:

Find the optimum design parameters $\mathbf{x}$ of a 3 PRR PPM in order to minimize the mass in motion of the mechanism and to maximize its regular shaped workspace subject to some design constraints, i.e., the inverse condition number of the kinematic Jacobian matrix and accuracy are to be higher than prescribed values throughout the manipulator workspace.

Mathematically, the problem can be written as:

$$
\begin{array}{cl}
\operatorname{minimize} & f_{1}(\mathbf{x})=m_{t} \\
\operatorname{maximize} & f_{2}(\mathbf{x})=R_{w}
\end{array}
$$

$$
\text { over } \quad \mathbf{x}=\left[\begin{array}{lllll}
R & r & L_{b} & r_{j} & r_{p}
\end{array}\right]^{T}
$$

$$
\begin{array}{ll}
\text { subject to }: & g_{1}: L_{b}+r \geq \frac{R}{2} \\
& g_{2}: 0<\rho_{i}<\sqrt{3} R \\
& g_{3}: \kappa^{-1}(\mathbf{J}) \geq 0.1 \\
& g_{4}: k_{x y}^{\text {min }} \geq \frac{F_{x, y}}{\sqrt{\delta x^{2}+\delta y^{2}}}=10^{6} \\
& g_{5}: k_{z}^{\text {min }} \geq \frac{F_{z}}{\delta z}=10^{5} \\
& g_{6}: k_{\phi_{z}}^{\text {min }} \geq \frac{\tau_{z}}{\delta \phi_{z}}=\frac{10}{\pi / 180} \\
& \mathbf{x}_{l b} \leq \mathbf{x} \leq \mathbf{x}_{u b}
\end{array}
$$

where $\mathbf{x}_{l b}$ and $\mathbf{x}_{u b}$ are the lower and upper bounds of $\mathbf{x}$, respectively.

\section{RESULTS AND DISCUSSIONS}

The multiobjective optimization problem (33) is solved by means of modeFRONTIER 28] and by using its built-in multiobjective optimization algorithms. MATLAB code is incorporated in order to analyze the system and to get the numerical values for the objective functions and constraints that are analyzed in modeFRONTIER for their optimality and feasibility. The lower and upper bounds of the design variables are given in Table 1. The manipulator is supposed to be built in steel with a density equal to $d=7850 \mathrm{~kg} / \mathrm{m}^{3}$ and a Young modulus equal to $E=210 \times 10^{9} \mathrm{~N} / \mathrm{m}^{2}$. 


\begin{tabular}{|l|c|c|c|c|c|}
\hline Design Variable & $R$ & $r$ & $L_{b}$ & $r_{j}$ & $r_{p}$ \\
\hline Lower Bound $(l b)[\mathrm{m}]$ & 0.5 & 0.5 & 0.5 & 0 & 0 \\
\hline Upper Bound $(u b)[\mathrm{m}]$ & 4 & 4 & 4 & 0.1 & 0.1 \\
\hline
\end{tabular}

Table 1: Lower and upper bounds of the design variables

For each design iteration, workspace limits are calculated based on the set of design parameters of the mechanism. Then, workspace discretization is performed with respect to its $x, y$ coordinates and with respect to the orientation angle $\phi$ of the moving platform. The constraints of the problem are evaluated at each grid point of the workspace.

\begin{tabular}{|l|c|}
\hline Scheduler & MOGA-II \\
\hline Number of iterations & 200 \\
\hline Directional cross-over probability & 0.5 \\
\hline Selection probability & 0.05 \\
\hline Mutation probability & 0.1 \\
\hline $\begin{array}{l}\text { DNA (DeoxyriboNucleic Acid) string } \\
\text { mutation ratio }\end{array}$ & 0.05 \\
\hline DOE algorithm & Sobol \\
\hline DOE number of designs & 30 \\
\hline Total number of iterations & $30 \times 200=6000$ \\
\hline
\end{tabular}

Table 2: modeFRONTIER algorithm parameters

A multiobjective genetic algorithm (MOGA) is used to obtain the Pareto frontier based on the mechanism mass and the workspace radius. modeFRONTIER scheduler and Design Of Experiments (DOE) parameters are given in Table 2. MATLAB is used to process and analyze the system for any individual of the current population (generated by the modeFRONTIER scheduler). Corresponding to each population set, MATLAB returns the output variables that are analyzed by modeFRONTIER for the feasible solutions according to the given constraints. At the end, the Pareto-optimal solutions are obtained from the generated feasible solutions.

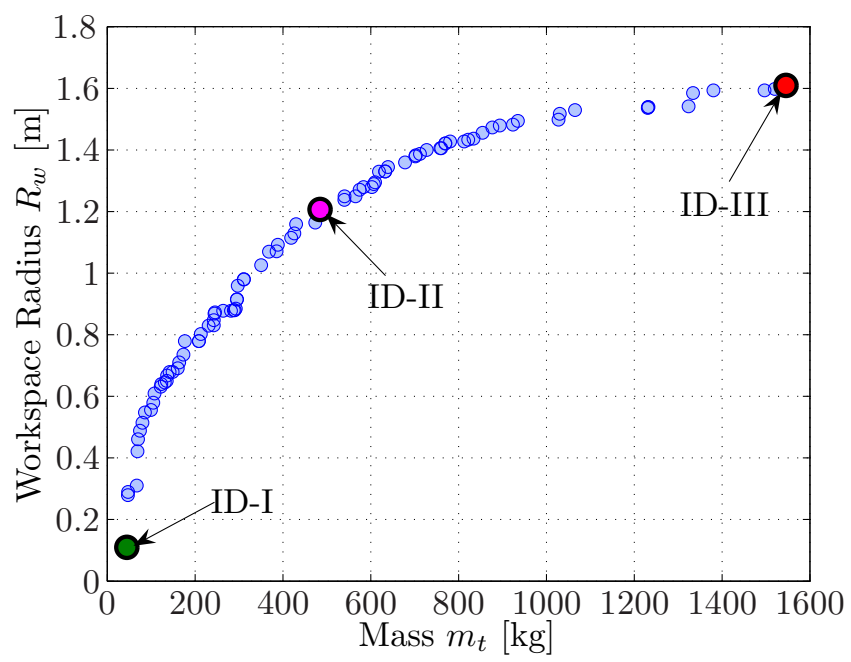

Figure 5: Pareto frontier for $3-\underline{P} R R$ optimization problem

The Pareto frontier is shown in Fig. 5 whereas the de- sign variables and the corresponding objective functions for two extreme and one intermediate Pareto optimal solutions, as shown in Fig. 5, are depicted in Table 3 .
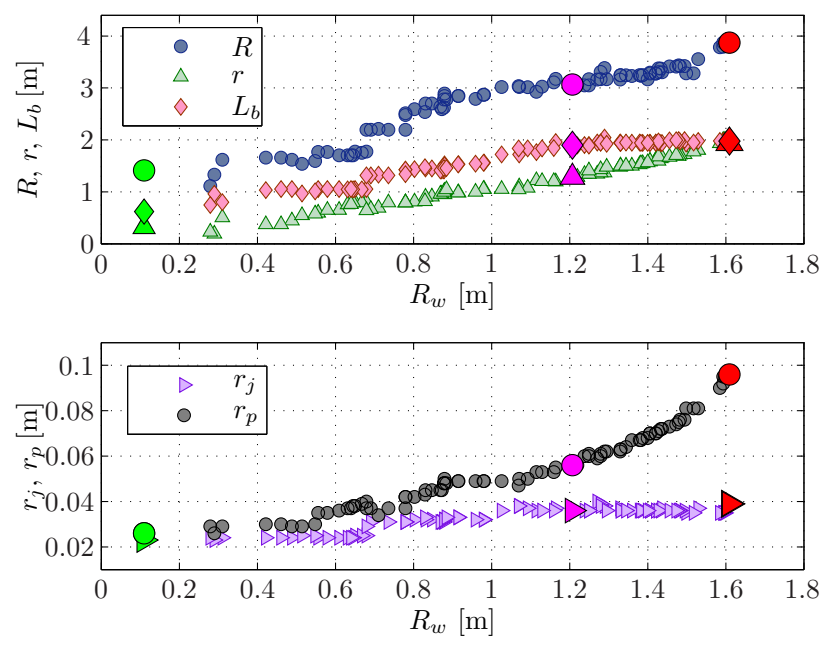

Figure 6: Design variables as a function of $R_{w}$ for the Pareto-optimal solutions

The designs associated with the three foregoing solutions are shown in Fig. 7 .

Figure 8 illustrates the variational trends as well as the inter-dependency between the objective functions and design variables by means of a scatter matrix. The lower triangular part of the matrix represents the correlation coefficients, $\xi$, whereas the upper one shows the corresponding scatter plots. The diagonal elements represent the probability density charts of each variable. The correlation coefficients vary from -1 to 1 . Two variables are strongly dependent when their correlation coefficient is close to 1 or -1 and independent when the latter is null.

Figure 8 shows that:

- Both objectives functions $m_{t}$ and $R_{w}$ are strongly dependent as their correlation coefficient is equal to 0.907 ;

- Both objectives functions $m_{t}$ and $R_{w}$ are strongly dependent on all design variables as all of the corresponding correlation coefficients are greater than 0.7;

- $R_{w}(\xi \geq 0.830)$ is slightly more dependent than $m_{t}(0.711 \leq \xi \leq 0.981)$ of the design variables;

Figure 6 illustrates the design variables $R, r, L_{b}, r_{j}$ and $r_{p}$ as a function of $R_{w}$ for the Pareto-optimal solutions. It is noteworthy that the higher $R_{w}$, the higher the design variables. It is apparent that the variations in variables $R, r, L_{b}$ and $r_{j}$ with respect to (w.r.t.) $R_{w}$ are almost linear whereas the variations in $r_{p}$ w.r.t. $R_{w}$ is rather quadratic. As a matter of fact, it should be due to the fact that the higher the size of the mechanism the higher the bending of the moving platform links whereas the intermediate links are mainly subjected to tension and compression. Finally, the three sets of design variables corresponding 


\begin{tabular}{|c|c|c|c|c|c|c|c|}
\hline \multirow{2}{*}{$\begin{array}{c}\text { Design } \\
\text { ID }\end{array}$} & \multicolumn{5}{|c|}{ Design Variables } & \multicolumn{2}{|c|}{ Objectives } \\
\cline { 2 - 8 } & $R[\mathrm{~m}]$ & $r[\mathrm{~m}]$ & $L_{b}[\mathrm{~m}]$ & $r_{j}[\mathrm{~m}]$ & $r_{p}[\mathrm{~m}]$ & $m_{t}[\mathrm{~kg}]$ & $R_{w}[\mathrm{~m}]$ \\
\hline I & 1.412 & 0.319 & 0.620 & 0.026 & 0.023 & 44.5 & 0.110 \\
\hline II & 3.066 & 1.283 & 1.896 & 0.036 & 0.056 & 484.8 & 1.207 \\
\hline III & 3.872 & 1.947 & 1.977 & 0.039 & 0.096 & 1545.6 & 1.609 \\
\hline
\end{tabular}

Table 3: Three Pareto optimal solutions

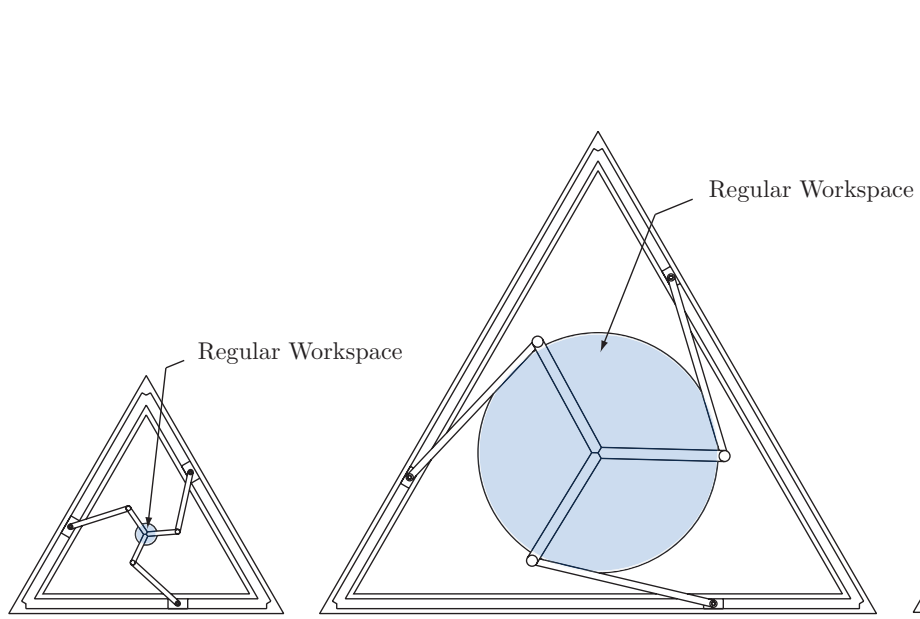

(a) ID-I

(b) ID-II

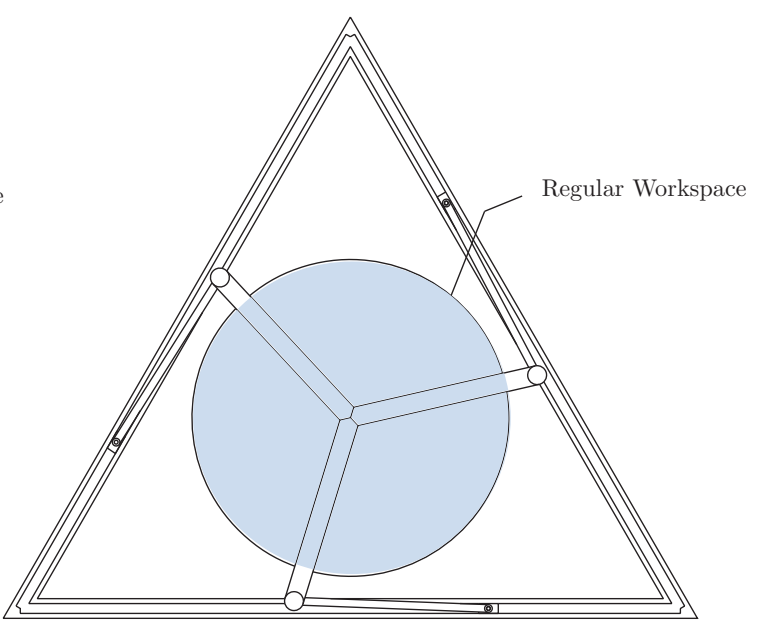

(c) ID-III

Figure 7: CAD Designs of three Pareto-optimal solutions

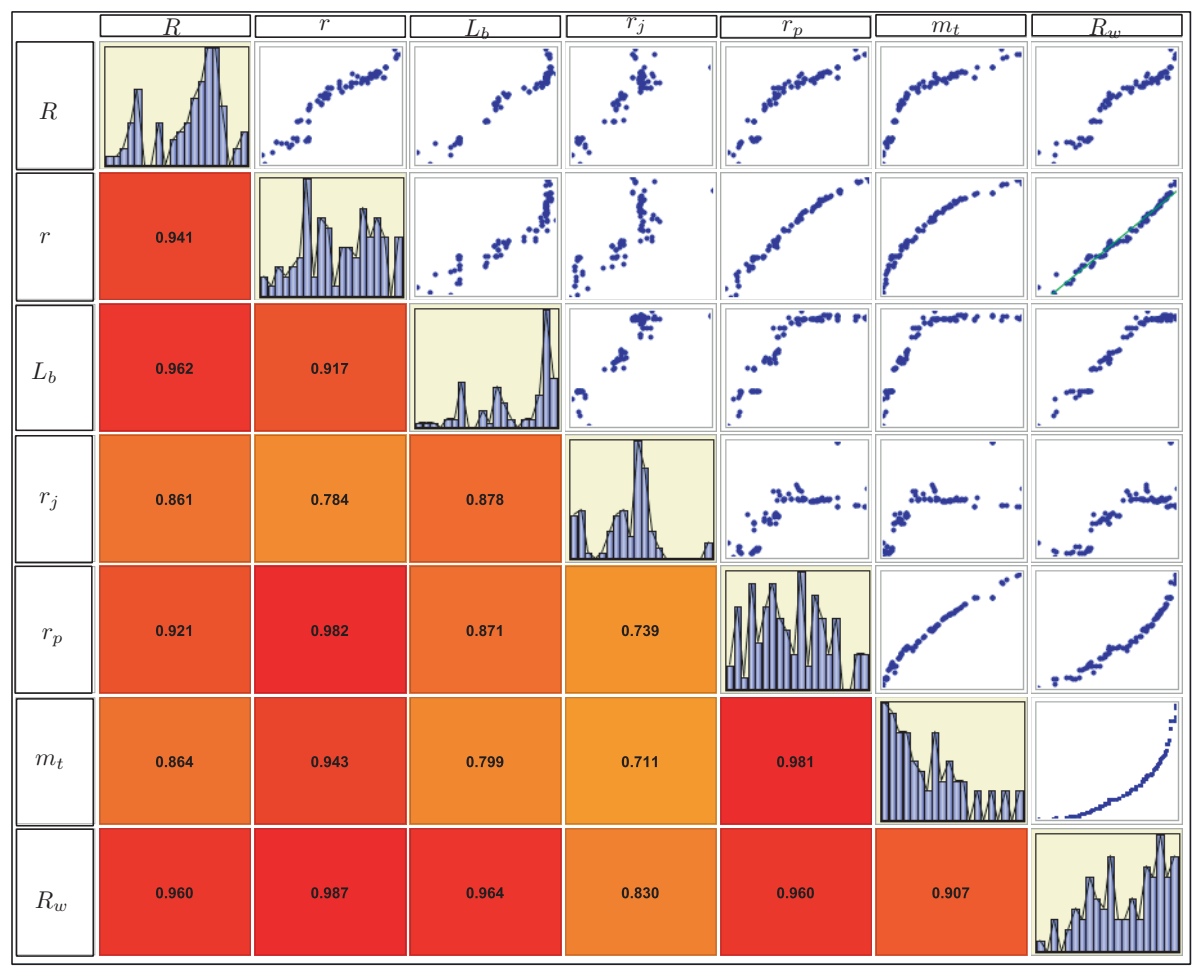

Figure 8: Scatter matrix illustrating the correlations between the objective functions and the design variables

to the Pareto-optimal solutions depicted in Fig. 5 are shown in Fig. 6 by means of the green, pink and red symbols.

\section{CONCLUSIONS}

In this paper, the problem of dimensional synthesis of parallel kinematics machines was addressed. A multi- objective design optimization problem was formulated in order to determine optimum structural and geometric parameters of any parallel kinematics machine. The proposed approach is similar to that used in 29. but we took into account the mass and the regular workspace instead of considering the entire volume of the manipulator. The proposed approach was applied 
to the optimum design of a three-degree-of-freedom planar parallel manipulator with the aim to minimize the mass in motion of the mechanism and to maximize its regular shaped workspace.

It is apparent that other performance indices can be used as constraints. However, they cannot necessarily be used as objective functions as the latter are usually formulated as a sum of an index over all the manipulator workspace. As another constraint, we could use the collisions between the legs of the manipulator as illustrated in [4].

Our future works will deal with the multiobjective design optimization and the comparison of 3-DOF planar parallel manipulators of different architectures as well as the optimization of the cross-section type of their links.

\section{REFERENCES}

[1] A. M. Hay and J. A. Snyman. Methodologies for the optimal design of parallel manipulators. International Journal for Numerical Methods in Engineering, 59(11):131-152, 2004.

[2] J. P. Merlet. Parallel Robots. Kluwer Academic Publishers, Norwell, MA, USA, 2006.

[3] Y. Lou, G. Liu, N. Chen, and Z. Li. Optimal design of parallel manipulators for maximum effective regular workspace. In Proceedings of the IEEE/RSJ International Conference on Intelligent Robots and Systems, pages 795-800, Alberta, 2005.

[4] Y. Lou, G. Liu, and Z. Li. Randomized optimal design of parallel manipulators. IEEE Transactions on Automation Science and Engineering, 5(2):223-233, 2008.

[5] E. Ottaviano and M. Ceccarelli. Workspace and optimal design of a pure translation parallel manipulator-tsai manipulator. Meccanica, 35(3):203-214, May 2000.

[6] E. Ottaviano and M. Ceccarelli. Optimal design of capaman (cassino parallel manipulator) with prescribed workspace. In $2^{\text {nd }}$ Workshop on Computational Kinematics KC2001, pages 3543, Seoul, South Korea, 2001.

[7] F. Hao and J.-P. Merlet. Multi-criteria optimal design of parallel manipulators based on interval analysis. Mechanism and Machine Theory, 40(2):157-171, 2005.

[8] M. Ceccarelli, G. Carbone, and E. Ottaviano. Multi criteria optimum design of manipulators. In Bulletin of the Polish Academy of Sciences Technical Sciences, volume 53, 2005.

[9] C. M. Gosselin and J. Angeles. The optimum kinematic design of a planar three-degree-offreedom parallel manipulator. ASME Journal of Mechanisms, Transmission and Automation in Design, 110:35-41, 1988.

[10] C. M. Gosselin and J. Angeles. The optimum kinematic design of a spherical three-degree-offreedom parallel manipulator. Journal of Mecha- nisms, Transmissions and Automation in Design, 111(2):202-207, 1989.

[11] H. H. Pham and I-M. Chen. Optimal synthesis for workspace and manipulability of parallel flexure mechanism. In Proceeding of the $11^{\text {th }}$ World Congress in Mechanism and Machine Science, pages 2069-2073, Tianjin, China, Apr. 1-4 2003.

[12] R. E. Stamper, L.-W. Tsai, and G. C. Walsh. Optimization of a three-dof translational platform for well-conditioned workspace. In Proceedings of the IEEE International Conference on Robotics and Automation, pages 3250-3255, New Mexico, 1997.

[13] M. Stock and K. Miller. Optimal kinematic design of spatial parallel manipulators: Application of linear delta robot. Transactions of the ASME, Journal of Mechanical Design, 125(2):292-301, 2003.

[14] C. Menon, R. Vertechy, M.C. Markot, and V. Parenti-Castelli. Geometrical optimization of parallel mechanisms based on natural frequency evaluation: application to a spherical mechanism for future space applications. IEEE Transactions on Robotics, 25(1):12-24, Feb 2009.

[15] H. Li, Z. Yang, and T. Huang. Dynamics and elasto-dynamics optimization of a 2-dof planar parallel pick and place robot with flexible links. Journal of Structural and Multidisciplinary Optimization, 38(2):195-204, 2009.

[16] M. Krefft and J. Hesselbach. Elastodynamic optimization of parallel kinematics. In Proceedings of the IEEE International Conference on Automation Science and Engineering, Edmonton, Canada, Aug 1-2 2005.

[17] D. Chablat and P. Wenger. Architecture optimization of a 3-dof parallel mechanism for machining applications, the orthoglide. IEEE Transactions On Robotics and Automation, 19(3):403410, 2003.

[18] D. Chablat, Ph. Wenger, S. Caro, and J. Angeles. The isoconditioning loci of planar 3-dof parallel manipulator. In Proceedings of DETC'2002, ASME Design Engineering Technical Conference, Montreal, Quebec, Canada, 29 Sep-2 Oct 2002.

[19] D. Chablat and P. Wenger. Working modes and aspects in fully parallel manipulators. In Proceedings of the IEEE International Conference on Robotics and Automation, pages 1964-1969, May 1998.

[20] C. M. Gosselin and J. P. Merlet. The direct kinematics of planar parallel manipulators: special architectures and number of solutions. Mechanism and Machine Theory, 29(8):1088-1097, 1994.

[21] A. Pashkevich, D. Chablat, and P. Wenger. Stiffness analysis of overconstrained parallel manipulators. Mechanism and Machine Theory, 44(5):966 - 982, 2009. 
[22] A. Pashkevich, D. Chablat, and P. Wenger. Design optimization of parallel manipulators for high-speed precision machining application. In $13^{\text {th }}$ IFAC Symposium on Information Control Problems in Manufacturing, Moscow, Russia, 3-5 June 2009.

[23] X. J. Liu, J. Wang, K. K. Oh, and J. Kim. A new approach to the design of a delta robot with a desired workspace. Journal of Intelligent and Robotic Systems, 39(2):209-225, Feb 2004.

[24] P. Wenger and D. Chablat. Kinematic analysis of a new parallel machine tool: The orthoglide. In Proceedings of the th International Symposium on Advances in Robot Kinematics, Portoroz, Slovenia, 2000.

[25] Z. Li. Geometrical consideration of robot kinematics singularities. The International Journal of Robotics and Automation, 5(3):139-145, 1990.

[26] B. Paden and S. Sastry. Optimal kinematic design of $6 \mathrm{r}$ manipulator. The International Journal of Robotics Research, 7(2):43-61, 1988.

[27] F. Ranjbaran, J. Angeles, M.A. GonzalezPalacios, and R. Patel. The mechanical design of a seven-axes manipulator with kinematic isotropy. ASME Journal of Intelligent and Robotic Systems, 14(1):21-41, 1995.

[28] ESTECO. modefrontier, version 4.0.3, 2008.

[29] O. Altuzarra, O. Salgado, A. Hernandez, and J. Angeles. Multiobjective optimum design of a symmetric parallel schnflies-motion generator. ASME Journal of Mechanical Design, 131(3):031002-1-031002-11, 2009. 